\title{
Life threatening interaction between tamoxifen and warfarin
}

\author{
P Tenni, D L Lalich, M J Byrne
}

Sir Charles Gairdner

Hospital, Queen Elizabeth

II Medical Centre,

Nedlands, Western

Australia 6009

P Tenni, BPHARM, senior

pharmacist (oncology)

D L Lalich, BPHARM, pharmacist

M J Byrne, MRCP, head of

department of oncology

Correspondence to: $\mathrm{Mr} \mathrm{P}$

Tenni, Department of

Pharmacy, Royal Perth

Hospital, Perth, Western

Australia 6001

BrMed f 1989;298:93
Lodwick et al reported a life threatening interaction between tamoxifen and warfarin. ${ }^{1}$ We report a retrospective study of similar cases in our hospital.

\section{Case reports}

A 43 year old woman started anticoagulant treatment with warfarin for a deep venous thrombosis. After a loading dose of $25 \mathrm{mg}$ over three days she was given $5 \mathrm{mg}$ daily, which maintained her prothrombin time at 19 seconds. Seven weeks later tamoxifen $40 \mathrm{mg}$ daily was added to her treatment. The next day her prothrombin time was 38 seconds, and eventually she was restabilised on $1 \mathrm{mg}$ warfarin daily to maintain a prothrombin time of 20-25 seconds.

This considerably reduced requirement for warfarin prompted a retrospective review of medical records at our hospital for the period 1981-6 to examine whether there might be an interaction between tamoxifen and warfarin. The records of women with breast cancer and a subsequent admission for a serious thromboembolism were examined. There were 18 such women: seven who had had deep venous thrombosis and 11 who had had a pulmonary embolus with probable deep venous thrombosis. Five of them were taking tamoxifen when they started taking warfarin. Two of them had complications after loading doses of warfarin: in one the prothrombin time after three daily doses of warfarin $(10 \mathrm{mg}, 10 \mathrm{mg}$, and $5 \mathrm{mg}$ ) rose to 50 seconds, and she developed a left subdural haematoma requiring warfarin to be withdrawn; in the other a similar loading dose regimen resulted in a prothrombin time of 49 seconds on day 7 , the patient developed severe haematuria, and her anticoagulant treatment was changed to phenindione. The three other patients taking tamoxifen required daily doses of $2 \mathrm{mg}, 2 \mathrm{mg}$, and $3 \mathrm{mg}$ to maintain appropriate prothrombin times.

The dose of warfarin that maintained appropriate prothrombin times in the 13 patients not taking tamoxifen varied from 4 to $10 \mathrm{mg}$ (mean $6.25 \mathrm{mg}$ ) daily. None of these patients had complications in the first month of treatment with warfarin.

\section{Comment}

Our observations suggest that women with breast cancer requiring warfarin need a lower dose if they are taking tamoxifen. The mechanism of the interaction between the two drugs is unclear, but protein binding and competition for metabolic pathways may both play a part. Both warfarin and tamoxifen are metabolised by the microsomal enzyme systems in the liver. Warfarin is a racemic mixture and the $(\mathrm{S})$ isomer is four to five times more physiologically active than the $(\mathbf{R})$ isomer. This more active (S) isomer is converted to the 7-hydroxy metabolite, which is inactive, by the cytochrome P450 enzyme system. ${ }^{2}$ Three metabolites have been detected in the serum of patients taking tamoxifen. Normally tamoxifen accounts for $36 \%$ of the drug and metabolites present, desmethyltamoxifen for $58 \%$, metabolite $\mathrm{Y}$ for $4 \%$, and 4-hydroxytamoxifen for $1 \cdot 5 \% .^{3}$ All of these metabolites have some affinity for the oestrogen receptor, but the affinity of the 4-hydroxy metabolite is 50-100 times greater than that of the parent drug. ${ }^{4}$ Accordingly, 4-hydroxytamoxifen is about 100 times more potent than tamoxifen in inhibiting the growth of MCF7 breast cancer cells in culture.

The hydroxylations are probably carried out by similar enzyme systems. In the case of warfarin competition for the enzymes may increase the concentration of the active parent drug and decrease the concentration of inactive metabolites. In the case of tamoxifen, however, the resultant changes in the amounts of metabolites present may have important implications for the activity of the drug. Altering the percentage of the 4-hydroxy metabolite present may have an effect on the response of the tumour.

The hazards of an increased pharmacological effect of warfarin and a theoretically decreased antitumour effect of tamoxifen make this a potentially serious drug interaction, which warrants further investigation.

1 Lodwick R, McConkey B, Brown AM, Beeley L. Life threatening interaction between tamoxifen and warfarin. Br Med f 1987:295:1141.

2 Kelly JG, O'Malley K. Clinical pharmacokinetics of oral anticoagulants. Clin Pharmacokinet 1979;4:1-15.

3 Furr BJA, Jordan VC. The pharmacology and clinical uses of tamoxifen. Pharmacol Ther 1984;25:127-205.

4 Fabian C, Tilzer L, Sternson L. Comparative binding affinities of tamoxifen, 4-hydroxytamoxifen and desmethyltamoxifen for estrogen receptors isolated from human breast carcinoma: correlation with blood levels in patients with metastatic breast cancer. Biopharm Drug Dispos 1981;3:381-90.

5 Coezy E, Borgna JL, Rochfort H. Tamoxifen and metabolites in MCF 7 cells: correlation between binding to estrogen receptor and inhibition of cell growth. Cancer Res 1982;42:317-23.

(Accepted 23 August 1988)

\section{Gastric explosion: a cautionary tale}

\section{J J Earnshaw, T K Keane}

City Hospital, Nottingham NG5 1PB

J J Earnshaw, FRCS, surgical registrar

T K Keane, FFARCS, anaesthetic registrar

Correspondence to: $\mathrm{Mr} \mathrm{J} \mathrm{J}$

Earnshaw, Derriford

Hospital, Plymouth

PL6 8DH

Br.Med f 1989;298:93-4
Gases in the colon will support combustion, and serious accidents have been reported when diathermy has been used for fulgurating colonic polyps. ${ }^{1}$ Cutting diathermy is often used for enterotomy in surgery on the stomach and small bowel and has been considered safe. We found, however, that under certain circumstances gases in the stomach may be explosive.

\section{Case report}

An 82 year old man with carcinoma of the gastric antrum proved by biopsy had a laparotomy under general anaesthesia. A rapid sequence induction technique was used to avoid gastric distension, and anaesthesia was maintained with $70 \%$ nitrous oxide, $30 \%$ oxygen, and $0.75 \%$ halothane. At laparotomy the stomach was moderately dilated owing to an extensive and inoperable carcinoma of the gastric antrum and pylorus. A large bore nasogastric tube was inserted and the stomach contents aspirated, though the tube soon became blocked by gastric debris. Cutting diathermy was used for the gastrotomy during a palliative antecolonic gastrojejunostomy. As the stomach was opened the gases within it ignited momentarily, an explosion was heard, and the scrub nurse and the theatre light were sprayed with gastric contents. The stomach was carefully inspected but seemed unharmed. The operation was continued, and the patient subsequently made an uneventful recovery.

\section{Comment}

We were not previously aware that gaseous gastric contents might support combustion, though one case has been reported. ${ }^{2}$ Initially we thought that 
anaesthetic gases had entered the stomach and caused this accident. Experiments have shown, however, that halothane of less than $7 \%$ concentration in $70 \%$ nitrous oxide with $30 \%$ oxygen cannot be ignited by a power source of $80-120 \mathrm{~J}$, which is much greater than that used in surgical diathermy $(10 \mathrm{~J}) .^{3}$ The presence of other gases, such as hydrogen and methane, would make an explosion possible. We postulate that fermentation can occur in an obstructed stomach and produce explosive mixtures.

We report this case to make surgeons aware that explosive gases may accumulate in the stomach when the gastric outlet is obstructed. Before operation we were unaware of the extent of the obstruction in this patient. We recommend that an obstructed stomach be completely emptied through a wide bore nasogastric tube either before or during surgery before cutting diathermy is used for gastrotomy. Alternatively, cutting diathermy could be avoided when the gastric outlet is obstructed.

We thank Mr J L Wilkins for permission to describe this case.

1 Bigard MA, Gaucher P, Lassalle C. Fatal colonic explosion during colonoscopic polypectomy. Gastroenterology 1979;77:1307-10.

2 Carroll KJ. Unusual explosion during electrosurgery. Br Med f 1964;ii: 1178 . 3 Churchill-Davidson HC. Flammable anaesthetic vapours. In: Wylie WD, Churchill-Davidson HC, eds. A practice of anaesthesia. 5th ed. London: Lloyd-Luke, 1984:240-4.

(Accepted 27 October 1988)

\section{Ethnic differences in birth statistics from central Birmingham}

\section{R Griffiths, M White, M Stonehouse}

Department of Community Medicine, Central Birmingham Health Authority, Birmingham B15 2TZ

Rod Griffiths, FFCM, director of health care policy

Martin White, MB, senior house officer

Mary Stonehouse, $\mathrm{MB}$, senior house officer

Correspondence to: Dr Griffiths.

Br.Med $\mathcal{f} 1989 ; 298: 94-5$ these findings.

\section{Methods and results}

During preparation of a health profile for Central Birmingham Health Authority ${ }^{1}$ a significantly raised postneonatal mortality was found among AfroCaribbean babies compared with European babies. A higher stillbirth rate and perinatal mortality among Asian babies and a lower stillbirth rate and perinatal mortality among Afro-Caribbean babies were also noted, as has been reported by nthers. ${ }^{2}$ We report

Our analysis was based on information collected for the Birmingham births register of the university department of social medicine. This is one of few sources of data on births in the United Kingdom where the ethnic origin of both parents is recorded. The sample comprised all births to parents resident in Central Birmingham Health Authority during 1982-4 inclusive (total number 9880). This was the largest sample obtainable: data from before 1982 were not readily available owing to changes in local authority boundaries, and data from 1985 onwards were incomplete at the time of the study. For each birth the outcome at one year was classified as stillbirth; death in the first, second, third, or fourth week; or death in the remainder of the year. Each birth was classified according to the ethnic group of both parents as European (white), Asian (Indian, Pakistani, or Bangladeshi), Afro-Caribbean, oriental, or mixed (when the parents were of different ethnic origin). The last two categories were combined to form "others" as the numbers were small. Statistical analysis was by the $\chi^{2}$ test with Yates's correction. Results for births among Asians and Afro-Caribbeans were independently compared with those among Europeans.

The table shows the results. The most notable finding was a significantly higher postneonatal mortality among Afro-Caribbean babies $\left(\chi^{2}=8.95\right.$, $\mathrm{p}<0.01)$. Causes of death in the postneonatal period were identified as far as possible by examining death certificates. Twenty seven babies (out of 56) were registered as having died of the sudden infant death syndrome. Congenital abnormalities accounted for 13 deaths, respiratory and other infections for five, accidental deaths for three, and other causes for three. Five files could not be located.
Number of births in Central Birmingham Health Authority in 1982-4 by outcome and ethnic group. Figures in parentheses are mortality/ 1000 births

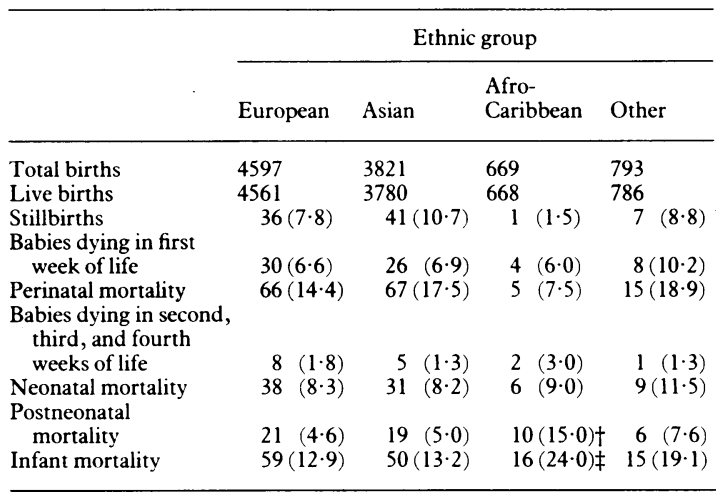

* Mortality expressed per 1000 live births except for stillbirth and perinatal mortality rates, which are expressed per 1000 total births. mortality rates, which are exp $\neq \chi^{2}=8 \cdot 95,0.001<\mathrm{p}<0.01$.
$\neq \chi^{2}=4 \cdot 71,0.02<\mathrm{p}<0.05$.

\section{Comment}

Increased postneonatal mortality and an increased incidence of the sudden infant death syndrome in AfroCaribbean babies have been reported in $\mathrm{America}^{4}$ but not in Britain. Data on ethnic origin are not routinely collected when births are registered in Britain, and it is therefore unlikely that such a difference would be detected unless looked for specifically. Other districts that we contacted could not give any indication of this trend. Reasons suggested for an increased postneonatal mortality among Afro-Caribbeans include a different socioeconomic distribution from that among other ethnic groups and a lower mean birth weight. ${ }^{4}$ In our sample 387 (58\%) Afro-Caribbean babies and 2363 (62\%) Asian babies were born to parents in social class IV, V, or unclassified compared with $1444(31 \%)$ European babies. Afro-Caribbean babies also had a different birthweight distribution, with $24(3 \cdot 6 \%)$ babies weighing less than $1500 \mathrm{~g}$ compared with 38 (1\%) Asian babies and $58(1 \cdot 3 \%)$ European babies. This high incidence of very low birth weight babies among Afro-Caribbeans agrees with the findings of another study in Birmingham. ${ }^{3}$

The low perinatal mortality among Afro-Caribbeans in our study is largely attributable to the low stillbirth rate, although this value was based on small numbers and was not significant. Other studies from Britain ${ }^{3}$ and America, ${ }^{5}$ however, have shown Afro-Caribbeans to have low stillbirth rates and neonatal mortality specific to birth weight. We report this high postneonatal mortality in Afro-Caribbeans in the hope that others will review their birth statistics to see whether ethnic differences in postneonatal mortality exist. 